\author{
Revista Venezolana de Gerencia (RVG) \\ Año 6. $N^{2} 13,2001,159-168$ \\ Universidad del Zulia (LUZ) • ISSN 1315-9984
}

\title{
La Nueva Política: Del Estado a las Redes*
}

\section{Gomá, Ricard**}

Antes de empezar fa conferencia queria hacer algunas anotaciones previas. En primer lugar y no podría ser de otro modo, de agradecimiento a todos ustedes y a la profesora Haydée Ochoa. En segundo lugar, a mis alumnos de doctorados quisiera pedirles por adelantado perdón porque no todo lo que van a oir va a ser nuevo, algunas de las cosas que voy a decir las hemos discutido en esta semana.

Creo que muchos de los conceptos que expondré pueden tener una cierta validez global pero nacen sobre todo de una reflexión y de un conocimiento empírico de lo que es la realidad especificamente europea y muy poco de lo que es su realidad nacional venezolana o latinoamericana en sentido más amplio, eso quiere decir que quizás muchos conceptos no tengan exactamente el mismo significado pero también quiere decir que puede existir el valor del contraste, el valor de poner en diálogo dos realidades y de poner en diálogo dos reflexiones y ver hasta que punto de ese diálogo puede salir una reflexión fértil y una réflexión enriquecedora tanto para ustedes como para mi.

Dichas estas anotaciones iniciales yo voy a estructurar mi intervención en tres apartados. Un primer apartado, previo a mi reflexión sobre la nueva política sobre las nuevas formas de goblerno y políticas públicas, voy hacer un repaso sobre los grandes y acelerados cambios estructurales que en el orden social, en el orden económico y en el orden cultural se han estado desarrollando en nuestras sociedades a escala global a lo largo de los últimos años, de la última década, creo que es importante empezar por allí, por esta reflexión, porque eso es lo que da consistencia a la argumentación que considera que la nueva política, que las nuevas formas de organizar los procesos de gobierno y de regulación püblica tienen actuaimente un fuerte soporte estructural y que en el ámbito de la política y del gobierno no puede quedar anciado en viejos paradigmas y anclado en viejas formas de realización cuando se mueve en un contexto de cambios económicos, sociales y culturales acelerados. Pero también empezar por alli significa reivindicar una vez más la autonomia de la politica, reivindicar que los grandes cambios estructurales hacen ineludible repensar y redefinir la esfera de la politica, pero que son los valores y la voluntades politicas las que al final van a determinar las orientaciones y los contenidos que en definitiva se van a plasmaren esa nueva política. Finalmente acabaré mi intervención de una manera breve, proponiendo de forma más propositiva algunos puntos que sencillamente enunciaré y si quieren ustedes los podemos

* Texto de la Conferencia dictada el día 1-2-2001 en el auditorio de la División de Estudios para Graduados de la Facultad de Ciencias Econónicas y Sociales de La Universidad del Zulia (LUZ). Invitado por el Doctorado en Ciencias Sociales, el Programa de Investigación sobre Politica y Administración subnacional, la Comisión para la creación de una Red de Politica y Administración Local (REPAL) y la Revista Venezolana de Gerencia.

** Investigador y Profesor de la Facultad de Ciencia Politica y de la Administración Pública de la Universidad Autónoma de Barcelona (España). E-mail: ricard.goma @uab.es 
dejar para un coloquio posterior, en clave ya algo más propositivo o normativo lo que a mi modo de ver deberían ser los puntos a fortalecer en ese contexto de nueva política.

Empiezo por estas nuevas grandes transformaciones o cambios en el orden social, cultural y económico que se han estado desarrollando yo creo que podemos hablar de tres grandes tendencias de tres grandes rasgos de fondo que definen de forma transversal a los cambios estructurales que se han estado desarrollando en la última década; esos tres rasgos serian: En primer lugar un incremento de la complejidad, de la complejidad en el orden social, económico y cultural. En segundo lugar una progresiva sustitución del espacio nacional por un nuevo eje, por una nueva configuración entre lo local y lo giobal y en tercer lugar un impacto social, económico y cultural potencialmente masivo de las nuevas tecnologias de la información y de la comunicación y especialmente lo que supone la red de redes "Internet".

En cuanto al incremento de la complejidad, el orden social de la modernización industrial fue un orden social de dicotomía y de rigideces en el terreno económico, en el orden económico existia fundamentalmente un gran eje de acumulación, basado en el trabajo en el sector industrial intensivo, en trabajo que determinaba la inclusión o la exclusión de grandes sectores populares en el proceso de modernización. En el orden social eso significaba un solo gran eje de estratificación que polarizaba a las sociedades en dos grandes agregados homogéneos de clases.

En el orden cultural, las identidades políticas, los valores colectivos y las culturas civicas en buena parte expresaban y reflejaban de forma directa y nítida las dicotomías económicas y sociales antes mencionadas. Todo ese escenario, todo ese contexto ha ido sufriendo una transformación creciente y podernos identificar un abanico de ejes de complejización en el orden social.

En lo económico, hoy podemos ya afirmar sin ningún género de dudas que el factor trabajo coexiste con otros factores muy poten- tes de acumulación, podemos citar todo lo que hoy implica la economia financiera, piensen ustedes que el valor diario de las transacciones financieras que se dan en los mercados financieros internacionales el valor diario equivale al Producto Nacional Bruto anual de la economía real de un pais como Francia, fijense ustedes por lo tanto la potencia de la economía financiera pero también el eje de acumulación que se define hoy en el terreno de la economia informacional y del conocimiento, cómo la información y el conocimiento se convierten a pasos forzados ya en uno de los factores predominantes de valor añadido en cualquier tipo de actividad económica.

Las estrategias de competitividad basadas en el factor coste y en la colocación de los productos en los mercados locales y regionales siguen siendo enormemente importantes, los análisis económicos más recientes nos ponen encima de la mesa una estadística que parecia infravalorada y es que 8 de cada $10 \mathrm{em}$ pleos están produciendo bienes y servicios que se consumen en mercados a escala local y regional, de allí la importancia de la escala local en la nueva economía, pero eso coexiste hoy con unas estrategias de competitividad basadas fundamentalmente en la calidad aportada por el conocimiento y que tienen una escala de colocación de bienes y productos en mercados fundamentalmente globales; sin salir del orden económico no podemos dejar de constatar como la organización fordista del trabajo coexiste hoy ya con un escenario de organización claramente postiordista del trabajo; es decir de desintegración de las jerarquías productivas de la antigua empresa industrial y la sustitución por escenarios de especialización flexibles desintegrados.

En el orden de lo social se ha producido una explosión de la diversidad y de la heterogeneidad en varios terrenos; en el terreno étnico cultural hoy no existe ninguna gran ciudad en el contexto europeo con menos de un $25 \%$ de su población conformadas por minorías étnico culturales. En el terreno de las estructuras familiares $y$ de las relaciones de género, la fa- 
miliar nuclear propia de la primera modernización industrial está dejando paso a una heterogeneidad muy importante en nuevas estructuras de convivencia primaria con el impacto que eso supone sobre las relaciones primarias, sobre las relaciones de género, sobre las relaciones de comunidad.

Otro terreno muy importante es ol terreno de la ampliación y de las nuevas formas percepción social sobre las diferentes fases y periodos del ciclo vital de las personas, con la redefinición y la ampliación de lo que significa la adolescencia, la juventud, la vejez etc., todo eso conforma un escenario, un panorama de heterogeneidad social como seguramente nunca se vivió en momento histórico anterior.

En el orden de lo cultural junto a procesos todavia inacabados e incompletos de modernización tradicional emergen y coexiste espacios de lo que hoy los grandes sociólogos están llamando la modernización reflexiva, que se traduce en actitudes políticas de alta volatilidad poco ancladas en las antiguas cos. mos visiones ideológicas en la emergencia de valores colectivos de tipo post-materialista y en la existencia de identidades múttiples y no excluyentes en cada uno de los individuos y de las personas de la sociedad, todo eso pues, conforma un escenario de nuevas complejidades que va a tener un impacto enorme y se va a traducir de alguna manera también en el orden de la política y más en concreto del gobiemo y de las políticas públicas.

Pero vamos al segundo gran eje transversal de cambios estructurales, lo que yo fomulé como la sustitución progresiva del espacio nacional por la nueva configuración local global por lo que los anglosajones han venido ya, en llamar la glocalización, como un término mucho más descriptivo de la realidad que el término de globalización, el eje local global; la glocalización como una nueva escala de expresión de los problemas sociales $y$ en consecuencia también de su tratamiento político, de su tratamiento colectivo.

Por una parte tenemos la escala global, en el tratamiento o mejor dicho en la percepción de los problemas, desde la conferencia sobre la biodiversidad y el medio ambiente que se celebró en Rio de Janeiro en 1992, pasando por la Conferencia sobre el Desarrollo Humano y Social de Copenhague en 1994, por la Conferencia sobre Población y Demografía en el Cairo, por la Conterencia de Mujeres y Relaciones de Género en China y la Conferencia sobre Habitad y Vivienda en Estambul, todas ellas bajo el paraguas organizativo de Naciones Unidas. Nunca antes en la humanidad habiamos tenido un inventario, un catálogo tan bien estructurado del carácter global de las problemáticas que afectan en el terreno ambiental, en el terreno del desarrollo social, en el terreno demográfico, en el terreno del género, en el terreno de la habitan y las ciudades. Por otro lado con ese incremento por lo menos de la conciencia y del conocimiento de esos problemas globales, coexiste una segunda realidad no menos importante y es que, es en el ámbito local, en el ámbito de las ciudades y de las regiones donde se expresan hoy, con enorme claridad los nuevos yacimientos de necesidades y de problemas sociales, directamente conectados con los ejes de complejidad social que anteriormente mencionaba, las fracturas étnicas los riesgos de exclusión derivados de la crisis de la familia nuclear, los nuevos problemas so. cio sanitarios, la segregación de los grupos sociales en el territorio urbano, el déficit en la función social de los sistemas de transporte colectivo, todo ello expresa también en la esfera de to local las nuevas complejidades sociales.

Finalmente, les comentaba como tercera formulación, el potencial de impacto que las tecnologías de la información y de la comunicación están ya desarrollando, a mi en. tender existe un enorme potencial de demow cratización del espacio informacional comunicativo y mediático gracias a la difusión de esas nuevas tecnologías; pero con ello coexiste también el riesgo de nuevas desigualdades informacionales y de nuevas fracturas digitales en nuestras sociedades. Esas nue vas tecnologias comportan un incremento muy considerable de los flujos de comunicación y de información, pero en la medida que 
estamos en un contexto de acceso social desigual a esos flujos y en la medida en que se está produciendo también una desigual capacidad de conversión de esa información en conocimiento por parte de unos u otros grupos sociales, eso entraña también determinados riesgos sociales. Las nuevas tecnologias tienen un enorme potencial de abolición de las distancias, de abolición de la geografla; pero a condición de una inclusión rápida de todos los territorios en la red sin excusiones territoriales, tienen un enorme potencial de flexibilldad en el tiempo y en el espacio de trabajo de cada uno, pueden incrementar, como era impensable hace poco tiempo, el grado de soberania individual sobre las propias condiciones de trabajo, pero pueden poner también en riesgo los elementos de socialización personal conectados a los entornos y a las comunidades tradicionales de trabajo. Finalmente las nuevas tecnologias tienen un enorme potencial para rehacer las formas de participación política y de acción colectiva, en el bien entendido que democracia electrónica la democracia directa, la posibilidad de participación a través de la red, no vaya en detrimento de los espacios de democracia deliberativa y de participación en comunidades, no solamente virtuales sino reales, de contacto humano.

Esos serian a mi entender y a grandes rasgos y con todo el esquematismo y la sintesis necesarias en este caso, los grandes ejes transversales de cambio estructural, ¿cómo todo eso se transfiere al ámbito del Gobierno y de las Politicas Públicas?, ¿Cómo el ámbito del Gobierno y de las Políticas Públicas se les adapta y se redefine en ese nuevo contexto? Voy a tratar también de reflexionar sobre la base de 4 grandes ejes transversales de redefinición del gobierno y de las políticas públicas. Por una parte lo que voy a llamar la nueva sustantividad, las nuevas orientaciones de fondo, de contenido, en muchas Políticas Públicas.

En segundo lugar, una nueva configuración temática de la política, en la reestructuración de las agendas del campo de lo que hoy es politizable y lo que no es politizable en el sentido profundo del término politizable; es decir, de regulable sobre la base de la colectivi. dad de la decisión pública.

En tercer lugar lo que voy a llamar las nuevas formas de acción colectivas, la rees. tructuración de los viejos actores y la emergencia de nuevos instrumentos y de nuevas formas de actuar colectivamente en el terreno de la política.

Finalmente la emergencia de mecanismos politicos de gestión de la complejidad que está tomando ya claramente la forma de redes la concepción de la gobernabilidad, de la governance reticular en forma de red como concepción predominante en el terreno no solamente teórico, sino creo yo ya empírico.

La nueva sustantividad: No es ningưn secreto que se están dando profundas reorientaciones de fondo en los principales contenidos de las políticas económicas, de las políticas sociales, de todo ese abanico de políticas que configuraron el espacio tradicional de la política en la sociedad industrial.

En el marco de la unión europea hay una convicción profunda de que en los últimos tiempos se ha producido una convulsión en todos los regímenes de bienestar que tradicionalmente se conformaron en los países de la unión; ahora bien, el resultado de esa convulsión no es ni mucho menos un resultado univo. co, es un resultado complejo, que encierra una gran diversidad de situaciones en función de cómo se han ido estableciendo las diferentes correlaciones de fuerza sociales y políticas en los diferentes territorios, en todo caso yo creo que no se puede afirmar que la resultante sea una lógica de desmantelamiento de las funciones políticas en el campo social y económico, sino una lógica de profunda reestructuración de esas funciones.

Por una parte se ha producido un fortalecimiento de algunas de las áreas tradicionales de política pública, la educación por ejemplo que se está convirtiendo en el eje fundamental de las políticas públicas en la medida en que la educación pública y de alta calidad se 
considera a medio y largo plazo el factor funda. mental de creación de valor económico y social en una sociedad; se fortalecen también los mecanismos de la protección social y de la negociación colectiva en el mercado de trabajo incluso, a lo largo de los últimos años podemos encontrar aportaciones de carácter innovador enormemente interesantes como por ejemplo la nueva agenda de lucha contra el desempleo en algunos países europeos que no pasa ya por la precarización del empleo, ni por la desregulación galopante del mercado de trabajo sino por estrategias innovadoras de reorganización y de reparto del tiempo de trabajo y por hacer aflorar nuevos yacimientos de empleo conectados a la satisfacción de nuevas necesidades sociales; pero todo eso coexiste en el contexto europeo hoy, con unos indices más elevados que antes de desigualdad, de pobreza relativa y de exclusión lo cual quiere decir que seguramente no ha existido una voluntad política suficiente para tratar desde el ámbito de lo público las nuevas necesidades sociales derivadas de la nueva sociedad compleja. Desde un punto de vista normativo ello me lleva a la conclusión que para la sustantividad de los contenidos de las políticas, la solución no es un repliegue de las funciones públicas de regulación económica y social, sino un nuevo salto adelante, un nuevo avance en esas funciones de regulación social y económica.

Ahora bien ello me lleva directamente al segundo punto de la reflexión, lo que yo formulaba anteriormente como las nuevas agendas del gobierno y de las políticas públicas, la apertura temática del gobiemo y de la política pública hacia realidades emergentes, hacia realidades nuevas, este también es un terreno complejo por una parte nos damos cuenta que el avance tecnológico ha producido la ineludible desimplicación política que significa por ejemplo la obsolescencia de determinados monopolios o cuasi-monopolios públicos en muchos mercados de la actividad económica e incluso de la nueva economia; por ejemplo, en las telecomunicaciones, pero nos damos cuenta también que existe ya de hecho un avance en el radio de acción de las políticas públicas cuando se plantea la regulación pública de internet, cuando se piantea la necesaria regulación pública de los avances biogenéticos en materia de seguridad alimentaria, en materia de prevención de riesgos ecológicos a de riesgos laborales; como también la intensificación y la profundización de la oferta pública en determinadas áreas tradicionales y la educación vuelven a servir aqui como el ejemplo a mi entender más paradigmático.

Junto a todo eso se produce también la emergencia de lo que se han venido en llamar hoy ya los derechos de la nueva generación o los derechos de cuarta generación; es decir ese conjunto de derechos que más allá de los derechos civiles, políticos y sociales que de alguna forma han ido avanzando en la humanidad a lo largo de las dos últimas décadas se sitúan ya en un nuevo escenario, derechos de cuarta generación que fundamentalmente implica una nueva oleada de derechos civiles, derechos de reconocimiento de las identidades colectivas y culturales de todo tipo de minoria y derechos en el terreno ambiental y ecológico; Asi pues por ejemplo las iniciativas politicas concretas del tratamiento de los flujos inmigratorios no sobre la base del origen de cada persona, sino de la residencia territorial de cada persona, el avance hacia politicas energéticas de desnuclearización y de sustitución de los modelos energéticos tradicionales por nuevos modelos energéticos, con energias limpias renovables y alternativas, la igualdad de derecho con independencia de las orientaciones culturales o sexuales de las personas o incluso el incremento de la soberanía personal sobre la vida de cada cual con el reconocimien to legal del derecho a la eutanasia, activa en algunos países, se sitúa hoy todo ello en el marco de esa nueva oleada de derechos y desde luego que esa nueva oleada de derechos enfrenta lo que el gran teórico Albert Hirschman bautizó como la retórica de la intransigencia, la retórica de la reactividad. Hirschman consideró que cada vez que en la humanidad sean introducidos nuevos derechos civiles, políticos y 
sociales, se han dado también retóricas reactivas sobre la base de tres grandes argumentos; el riegos, la sutilidad y la perversión.

El riesgo, es decir; poner en cuestión los avances obtenidos desde ese momento, la sutilidad, considerar que los cambios no son relevantes y la perversión, considerar que lo que vendrá será siempre peor de lo que tenemos. Desde una perspectiva actual creo que es necesario combatir esa retórica de la reactividad que se está ya produciendo con otra retórica del cambio y de la transformación, que considera que la urgencia, que la posibilidad y que de alguna manera la ineludibilidad del cambio hacen necesaria ese avance también en el terreno de los derechos.

En tercer lugar comentaba la aparición de nuevas redes de actores y de nuevas formas de acción, de acción colectiva, no descubro nada cuando afirmo que los partidos de masa que vehicularon la representación politica de la sociedad durante las décadas centrales de la modernidad industrial que ofrecian recursos de identidad globales a la sociedad y que tenían un carácter socializador e integrativo han dejado de existir como tales, eran partidos políticos que además de alguna forma satelizaban todas las otras formas de organización colectiva en la sociedad, que satelizaban incluso al movimiento obrero en un intercambio de canalización de demandas por apoyo electoral de amplias capas populares.

En el contexto europeo la crisis de esa forma de representación política y de articulación colectiva tiene seguramente su punto de arranque en lo que fueron las revoluciones del año 68 en varias cludades europeas Paris, en Praga, etc., en ese momento surgen unos nuevos movimientos sociales que ponen sobre la mesa ya la concertación de que por primera vez entre el siglo $\mathrm{X}$ y el siglo XX la izquierda clásica pierde el liderazgo como actor portador de un proyecto de cambio social en beneficio de otros sectores emergentes que no son comprendidos desde los parámetros de ese progreso clásico que de alguna manera configuró la izquierda tradicional; pero ese esquema de unos partidos de masas en crisis y unos nuevos movimientos sociales que nacen a partir del 68 también hoy empieza a estar superado por una tercera generación de formas de acción colectiva. Una tercera generación que pasa por dos grandes ejes. Por una parte, los partidos políticos abandonan claramente cualquier pretensión de socialización política, no son aceptados como tales por las sociedades civiles más avanzadas y reflexivas pero de la misma manera que se afirma esto afirmo también que esos partidos solamente pueden sobrevivir si reelaboran su identidad y su contenido programático para hacerlos expresión instjtucional de las nuevas contradicciones sociales. Yo no soy de los que cree que desaparecen los ejes de conflictos tradicionales, no soy de los que cree que dejan de tener sentido, conceptos como la derecha o la izquierda pero también cree que tanto sentido como eso hoy tiene la dicotomía entre lo nuevo y lo viejo, tanto en la derecha como en la izquierda.

Por otra parte se produce una convulsión paralela en el campo de los nuevos movimientos sociales, en su organización en su mortologia en los temas que tratan esos nuevos movimientos sociales y en el repertorio de formas de acción de acción colectiva, de tal manera que hoy, incluso la teoría sociológica más avanzada, está dejando de hablar de movimientos sociales para hablar de comunidades de acción colectiva que se estructuran en forma de redes de acción colectiva que se introducen en las nuevas formas de gobernabilidad pasando de la simple resistencia antes los antiguos aparatos e instituciones estatales, a una creatividad mucho más propositiva y por lo tanto que aporta mucho más para hacer avanzar la governance hacia un escenario de más democratización más abierto, más participativo.

Finaimente llego al cuarto concepto transversal de la nueva política que es el concepto de red, la red como la estructuración predominante ya en el campo empírico de las nuevas formas de organización de los procesos de gobierno, entendiendo por la red; esa estructuración de la política en donde todos los actores 
son conscientes de las interdependencias, en donde los actores son conscientes de la noexistencia de centralidades jerárquicas fuertes y son conscientes también de la necesidad de dar una cierta institucionalización a esas redes; redes que pueden ser muy diversas, pero que se están desplazando progresivamente de los elementos más tradicionales, es decir; de los recursos jurídicos y de los recursos y el manejo de recursos económicos hacia un campo donde lo cognitivo, donde lo simbólico, donde lo cultural, donde lo intangible pasa a tener una enorme relevancia en el contexto de esas redes. Creo que es necesario distinguir entre hacer una cierta distinción aunque después en la realidad todo está fuertemente entremezclado, entre las redes de participación social y las redes públicas a múltiples niveles territoriales.

Las redes de participación social serian las redes que se conforman a partir de la consideración de que no existe en un solo territorio o en solo nivel de gobierno un solo actor público o privado con los niveles de información y de manejo de recursos cognitivos, informacionales, económicos suficientes como para dar respuesta a las nuevas complejidades sociales y eso por lo tanto determina el inicio de la configuración de una red para tratar y dar una determinada orientación en la resolución de esas problemáticas. Creo que es muy importante reflexionar sobre el hecho de no solamente la erosión y la degradación de los antiguos consensos políticos que de alguna forma ofrecian la clave de resolución de todo los problemaso por lo menos eso pretendían, sino también la erosión de muchas de las certezas técnicas que se pusieron a disposición de la resolución de esos problemas, hoy desde el punto de vista técnico no predominan las certezas, predo. minan las incertidumbres en el tratamiento de los nuevos problemas sociales y cuando se produce un incremento de las incertidumbres técnicas y simultáneamente se produce un incremento también de las complejldades polittcas y de la erosión de las activos consensos, los procesos de formulación de políticas públicas y de gobierno entendidos como procesos de programación racional, dejan de tener sentido y es necesario avanzar hacia escenarios en donde los conceptos clave pasan a ser la experimentación, la negociación y el aprendizaje sobre todo el aprendizaje, el aprendizaje social.

Seguramente durante muchas décadas desde las perspectivas más racionales de entender el gobierno, se consideró que existía algo así como una relación inversamente proporcional entre la participación y la eficiencia en la resolución de los problemas públicos, de tal forma que el incremento de la complejidad participativa iba en detrimento de la eficiencia, de las soluciones técnicas que se proponian por parte de aquelios colectivos que de alguna forma monopolizaban esos conocimientos técnicos, hoy en un contexto radicalmente diferente de incentidumbres técnicas y de incertidumbres politicas, esa relación inversamente proporcional entre eficiencia y participación da un vuelco, da un giro de 180 grado de tal forma que seguramente será hoy ya una correlación directamente proporcional entre la eficacia en la resolución de los problemas y el grado de participación social en la resolución de esos problemas, de tal manera que un incremento de la eficacia va acompañado de un incremento tamblén de las formas de participación social y politica, eso síbien instrumentadas A través de nuevos instrumentos y nuevas formas de participación, que deben dar prioridad a una reflexión sincerada y deliberada de los problemas que deben ser pluralistas, que deben atender a los diferentes intereses organizados; pero que también deben dar cabida a eso que los sociólogos llaman ya hoy el nuevo individualismo democrático, es decir la capacidad de asunción de responsabilidades personales en el espacio público o como llaman otros autores el incremento del capital social de las redes de confianza y de reciprocidad e interindividual en la solución de problemas.

También tenemos que hacer referencia a 10 que se llama las redes públicas de gobiernos de múltiples niveles territoriales; si antes constatábamos la imposibilidad de que un solo 
actor público o privado pudiera resolver problemas complejos en un solo territorio tenemos que hacer una comparación en paralelo y es que desde un solo nivel territorial diticilmente van a poder resolverse los nuevos problemas complejos y eso implica encontrar, experimentar y en definitiva aprender sobre lo que pueden ser las nuevas formas de gestión política de carácter global, de carácter supranacional pero también de carácter territorializado de carácter regional y local.

En el contexto de la Unión Europea se ha producido a lo largo de la década de los noventa un avance impresionante en la europeización de las políticas públicas de tal forma que yo me atrevo hoy a afimar que la Unión europea es ya, en el terreno de las políticas públicas, un estado federal, no en el terreno de la institucionalidad ahi hay muchisima más debilidad pero sí en el terreno del gobiemo y de las políticas públicas, ¿Cómo podemos, sino contextualizar a una Unión que monopoliza prácticamente, 0 sin prácticamente que monopoliza la capacidad de diseño de políticas tan importantes como las monetarias, como la agricola, como la de cohesión interregional, como la de regulación ambiental, como la de regulación laboral, como la de ciencia y tecnología o como la de comercio exterior; alli por lo tanto tenemos unas fórmulas de federalización supranacional del gobiemo y de las políticas públicas que sitúan la escala territorial, para dar respuesta a esos problemas claramente y sin ningún complejo, por encima del Estado $\mathrm{Na}-$ ción, eso si, desde una voluntad de articulación tendencialmente simétrica entre los Estados $\mathrm{Na}$ ciones y no desde un nuevo eje de dependencias de unos estados sobre otros.

Hablaba también sobre la territorialización, de la necesidad de fortalecimiento, por lo tanto de los niveles locales y regionales de elaboración de políticas públicas. En el contexto europeo el desarrollo del Estado de bienestar en sus formas más clásicas infravaloró claramente las aportaciones que los ámbitos locales y territoriales podían jugar en el incremento de los niveles de bienestar y calidad de vida, hubo determinados países en los cuales los ni- veles territoriales y locales se convirtieron eso sí en una gran agencia prestadora de servicios, pero sin posibilidad de pensar y de reflexionar sobre el contenido de los servicios que estaban prestando porque la reflexión sobre los contenidos se daba en escalas superiores, hubo otros Estados que si situaron a los poderes locales capacidades de autonomía política, pero en cambio no los dotaron de los recursos suficientes para apoyar esas capacidades de elaboración de políticas; ambos escenarios fueron insatisfactorios.

También asistimos en los últimos años a un abanico de pactos en los diferentes Estado opción tendente que viene a incrementar las capacidades de gestión de recursos por parte de los poderes territoriales o bien incrementar las capacidades de diseño de políticas públicas por parte de esos niveles territoriales; les voy a poner dos ejemplos, hace solamente 20 años del conjunto del gasto público de los países de la Unión Europea el $90 \%$ se gestionaba y se gastaba directamente en el nivel nacional y solamente un $10 \%$ en los niveles territoriales hoy esa distribución está en 60-40, $60 \%$ en el nivel nacional y $40 \%$ en los niveles locales lo cual significa en dos décadas un salto enorme del 10 al $40 \%$, eso en el terreno de la capacidad de gestión de recursos pero tamblén en el terreno de la capacidad de diseño de políticas públicas; hoy en el contexto europeo cuando se habla de referentes innovadores en el terreno del gobierno y las políticas püblicas muy pocas veces se hace referencia a Estados, a Naciones y en cambio en la mayoria de las veces se hace referencia a gobiernos locales y ciudades; se habla de Barcelona, de Milán, de Ámsterdam, como los espacios donde se han experimentado las experiencias más interesantes y más innovadoras en el terreno del gobierno y de las políticas publicas, algo que era impensable hace solamente 2006 años.

Finalmente los niveles locales y los niveles globales deben articularse, no pueden desarrollarse de manera segmentada sin ningún tipo de relación hay por lo tanto un reto planteado muy importante que es el fortaleci- 
miento de las relaciones interinstitucionales, ahora bien ihacia que tipo de relaciones interinstitucionales o intergubernamentales se debe avanzar?; yo creo que debe ser un tipo de relaciones interinstitucionales que abandonen el concepto de jerarquía, es decir los niveles territoriales superiores no pueden considerarse jerárquicamente superiores a los niveles territoriales inferiores, sino que debe ser un modelo de relaciones institucionales basados en dos premisas: En la autonomia es decir; en la posibilidad de un diálogo paritario de un diálogo de igual a igual, de un dialogo simétrico entre los diferentes niveles territoriales y también de una relaciones de confluencia de interdependencia de lo que algunos autores llaman el federalismo reticular o el federalismo complejo es decir desarrollar las culturas políticas que permiten la confluencia no jerarquizada sino simétrica de diferentes niveles territoriales de gobierno en cada uno de los sectores de política pública como mejor garantía para la resolución de los problemas sociales.

Voy a terminar mi exposición para no cansarles excesivamente proponiendo una especie de decálogo de puntos concretos o de proposiciones muchas de las cuales derivadas de las reflexiones que yo he desarrollada hasta ahora y que a mi entender pueden dar un cierto contenido, una cierta orientación a esa nueva politica y que en cualquier caso pongo encima de la mesa para su posible reflexión posterior.

En primer lugar; la necesidad de un cambio muy importante en la cultura política y en la cultura organizativa no solamente de los ciudadanos sino del conjunto de los representantes políticos y de los empleados públicos, un cambio en la cultura politica y organizativa que pase por reforzar la institucionalidad, pero por reforzar la institucionalidad bajo unos parámetros de más flexibilidad de más orientación hacia la resolución de los problemas públicos.

En segundo lugar situar en el eje de las agendas, de intervención de las agendas de gobierno más tradicionales el tema de la educación de calidad, de la educación universal como la mejor garantia, no solamente para so- ciedades competitivas sino también para sociedades cohesionadas socialmente.

En tercer lugar; una apuesta clara y decidida hacia la apertura del gobierno hacia los nuevos derechos que yo antes comentaba; a los nuevos derechos civiles, hacia los nuevos derechos de reconocimiento de nuevas identidades culturales y hacia los nuevos derechos ecológicos.

En cuarto lugar una apuesta por fortalecer las esferas de gobierno de proximidad en un marco de relaciones institucionales basadas en la simetría y no en la jerarquiaEn algún momento se hizo circular el eslogan de que era necesario pensar globalmente para actuar localmente; yo creo que es necesario darle la vuelta a ese eslogan y tenemos que afirmar hoy que es necesario pensar localmente para actuar globalmente, pensar localmente para fortalecer la resolución local de problemas y actuar globalmente algo que hoy es perfectamente posible gracias al avance de las tecnologías de la información y de la comunicación.

Un siguiente punto pasaría por la reestructuración de los gobiernos globales tanto por avanzar en los procesos de federalización regional; yo soy un enorme convencido de la necesidad de seguir avanzando en esos procesos de integración regional en el que la Unión Europea creo que puede ser el ejemplo más avanzado, pero también en reestructurar otros gobiernos más globales yo creo que hoy es una necesidad imperiosa a mi modo de very aunque esta puede ser una afirmación muy fuerte, abolir al Fondo Monetario Internacional y abolir al Banco Mundial, y sustituirlos por una nueva agencia de desarrollo económico y social bajo el paraguas de Naciones Unidas.

Finalmente, fortalecer las dinámicas de participación social, fortalecer las dinámicas de participación social en un doble sentido; en el sentido de que eso va a provocar o tiene que provocar un for- talecimiento de los valores civicos de la impregnación de los valores democráticos en un sentido profundo, pero también por su fusionalidad, porque eso va a provocar también mayores dosis de eficacia y 
eficiencia en la resolución de los problemas. $Y$ finalmente algo que cada vez es más posible también, el aprendizaje, el aprendizaje transnacional, el aprendizaje de experiencias entre diferentes programas que se da en diferentes ciudades, en diferentes te- rritorio, en diferentes países del mundo, $y$ eso es algo muy importante porque de alguna manera puede hacer ver que lo que en un determinado lugar, $y$ en un determinado momento puede ser un desideratum, puede ser incluso una utopía, en ese mismo momento y en otro lugar ya es una realidad palpable y eso puede ser también un elemento muy importante para dotar de lo que Gramsci llamaba el optimismo de la razón; es decir el reforzamiento de la idea de que a través de la acción colectiva, a través de la acción política, a través de formas democráticas y participativas de gobierno es posible ir mejorando los niveles de desarrollo humano, de desarrollo social, de bienestar, de calidad de vida para todas las personas. Bien, hasta aqui mis reflexiones en la conferencia, muchas gracias por prestar atención. 